\title{
Using A Minimum Tile Path For Plant Transformations Encom- passing the Entire Soybean Genome
}

\author{
Hidayat Ullah ${ }^{1,2}$, Mohammed Jasim ${ }^{1,3}$ and David A Lightfoot ${ }^{1 *}$
}

1 Department of Plant, Soil and Agricultural Systems, Genomics and Biotechnology Core Facility, Centre for Excellence, The Illinois Soybean Centre, Southern Illinois University, Carbondale IL, 62901, USA; ${ }^{2}$ Faculty of Agriculture, Abdul Wali Khan University, Mardan, Khyber Pakhtunkhwa, Pakistan; ${ }^{3}$ Department of Biotechnology, Al-Anbar University, Ranadi, Iraq

Received: October 10, 2011 / Accepted: January 20, 2012

\begin{abstract}
Genomes like Glycine max (soybean) that have been highly conserved following increases in ploidy represent a frontier for genome analysis. Many soybean QTL analyzed to date have been composed of gene clusters each with contributing a portion of the trait rather than alleles of single genes. At the Soybean Genome Database (SoyGD) http://soybeangenome.siu.edu the genome browser that integrates and served the publicly available soybean physical map, BAC fingerprint database and genetic map associated genomic data shows a minimum tile of transformation ready BIBAC-like clones in pCLD04541 ( $p$ V1; oriV; tra; bom). Sequence resources made available through the DOE genome sequencing project have allowed the minimum tile to be revised and new functional analyses to be made. There are 3,840 MTP clones that appeared to encompass $90 \%$ of the genome (see http://soybeangenome.siu.edu/cgi-bin/gbrowse/BES_scaffolds). The BIBAC-like clones (tetR) from E. coli DH1O B were transferred en masse to Agrobacterium tumefaciens by triparental matings with EHA105 (rifR) mediated by PRK2013 (oriP) in DH1OB (kanR) in $\mathbf{3 8 4}$ well plates. Although not necessary the extra helper plasmid boosted efficiency 10 fold. Individual $A$. tumefaciens rifampicin and tetracyclin resistant strains were used for transformation of Arabidopsis thaliana flowers in $\mathbf{3 8 4}$ well arrays. Initially kanamycin selection was used to isolate transgenic plants. Because the BACs were already tetR the recA mutants of $A$. tumefaciens could not be used (Tn3 insertions). Consequent to this and partial transconjugation events only some inserts are transferred completely while
\end{abstract}

other transformed lines contain a substitution series of deleted inserts anchored on the Ti-left border (LB). These are maintained as kanR mixtures of seed. Phenotypes found for lines transgenic for particular BACs that were repeated include seed composition (protein, oil), development (growth, senescence) and disease resistance (suddean death syndrome (SDS) and soybean cyst nematode (SCN).

\section{Introduction}

Legume crops are particularly important for all cropping systems, world over, due to their ability to support symbiotic nitrogen fixation, a key to sustainable crop production and reduced carbon emission (Lightfoot, 2008). Among legumes, however, soybean (Glycine max) has a special position as a major source of increased production in the common grass-legume rotation. The soybean crop has high seed protein content ( 40\%); good seed oil content ( $\sim 20 \%$ ) and is broadly tolerant to many diseases and stresses. In the past, attempts for genetic improvement to increase soybean seed yield largely relied on selection from the existing variability among cultivars. Often selection is focused on increased resistance to various diseases to avoid yield losses due to these diseases.

The 'Forrest' variety of soybean was so named for the abilities of a southern Civil War General to arrange a defense (Hartwig and Epps, 1973). The cultivar 'Forrest' and other Forrest-derived lines like 'Hartwig' and 'Ina' have saved US growers billions of 
dollars in crop losses due to resistances programmed into the genome of Forrest cultivar (Wrather et al., 1997; 2003). Moreover, since Forrest grows well in the north-south transition zone, breeders have used this cultivar as a bridge to introduce a great deal of quantitative genetic variation from the southern to the northern US gene pool (Lightfoot, 2008).

Over the past decade, investment in Forrest genomics resulted in the development of the many resources for $r$ genomics research (Lightfoot, 2008). Resources included: (i) a genetic map; (ii) three RIL populations (96>n>975); (iii) 200 NILs; (iv) 115,220 BACs and BIBACs; (v) a physical map; (vi) 4 different minimum tiling path (MTP) sets; (vii) 25,123 BAC end sequences (BES) that encompass $18.5 \mathrm{Mbp}$ spaced out from the MTPs; (viii) a map of 2,408 regions each found at a single position in the genome and 2,104 regions found in 2 or 4 similar copies at different genomic locations (each of $>150 \mathrm{kbp}$ ); (ix) a map of homoeologous regions among both sets of regions; $(x)$ a set of transcript abundance measurements that address biotic stress resistance; (xi) methods for transformation; (xii) methods for RNAi; (xiii) a TILLING resource for directed mutant isolation and (xiv) analyses of conserved synteny with other sequenced genomes. Data on the Forrest genome and resources are provided to the scientific community through SoyGD, LIS, Soybase and GenBank (Wu et al., 2004a,b; Shultz et al., 2006a,b; 2007a,b; Saini et al., 2008).

In combining desired characters, the structure of loci and even chromosomes appears to be pivotal to the special qualities of the Forrest genome (Ruben et al., 2006; Afzal et al., 2009; Karangula et al., 2009). Genes underlying many quantitative and qualitative loci are targeted for isolation in the laboratories of the worldwide collaborating groups. What was missing until recently (Hamilton et al., 1997) was a methods to test the function of BACs or genes within BACs in planta. Transformation with large insert clones has become a limiting need. Here that earlier work was extended to BACs from soybean.

In preliminary work genes isolated from Forrest-derived BACs were used for transformation of $A$ thaliana by the floral drop method (Lightfoot and Ullah, 2009). BACs used included those encoding candidates for resistance to nematode (Rhg 4 and $r$ h 1 ), resistance to Phytophthora sojae (Rps5), resistance to Pseudomonas syringae (Rps 1 ) and resistance to Fusarium virguliforme (Rfs2). These resources also assisted in the genomic analysis of soybean nodulation ( $\mathrm{GmNark}$ and $\mathrm{GmNod}$ ). Additional loci for seed yield, seed composition as well as resistances to 3 biotic stresses, 4 fungal species and 3 nematode species have been identified (unpublished reports). Here is reported for the first time the transformation of the entire soybean genome into $A$. thaliana as the minimum tile path of BACs from the Forrest physical map and genome sequence.

\section{Materials and Methods}

There are 3,840 MTP clones that appeared to encompass $90 \%$ of the genome (see http://soybeangenome.siu.edu/cgibin/gbrowse/BES_scaffolds; Shultz et al., 2006a,b; 2007a,b). The BIBAC-like clones from $E$. coli $\mathrm{DH} 1 \mathrm{OB}$ were transferred en masse to $A$. tumefaciens EHA 105 by triparental matings (Figure 1) mediated by pRK2013 in 384 well plates on solid TY media (no selection). Individual A. tumefaciens strains were selected for rif and kan resistance twice, once on solid media then in liquid media.

The rif/tetR strains were used for transformation of $A$. thaliana flowers following 2 fold dilution with $0.5 \mathrm{MS} 0.005 \%(\mathrm{w} / \mathrm{v})$ Sillwet50 and 384 clone-to-pot arrays (Figure 2). Kanamycin selection with Sillwet50 adjuvant was used to isolate transgenic plants by applications on three occasions, 7 days apart, to T1 lines $(7,14$ and $21 \mathrm{dag})$. Seed were collected in eppendorf tubes at $\mathrm{T} 1$ to ease reuse and distribution. T2 seed were selected for KanR but collected into 96 well plates to allow for robotic methods of analysis.

Transformed plants and their progenitor strains were tested with the appropriate BES and marker amplicons by PCR to assess the extent of insert stability in A. tumefaciens and the proportion of the insert transferred to the plants (Figure 3).

Phenotypes tested for among transgenic plants included altered flower color, disease resistances, seed yield, seed protein, seed oil, seed isoflavone content, nitrogen use efficiency, water use efficiency and herbicide resistances..

\section{Results}

\section{Assessing the Efficiency of Transformation}

PCR amplicons showed about $50 \%$ of KanR plants had complete inserts and $50 \%$ had deletion derivatives (Figure 3 ). The deletion derivatives appeared to derive from instability in the A. tumefaciens rather than the plant. This might be expected since the KanR derived from nptll in pCLD04541 is between the left

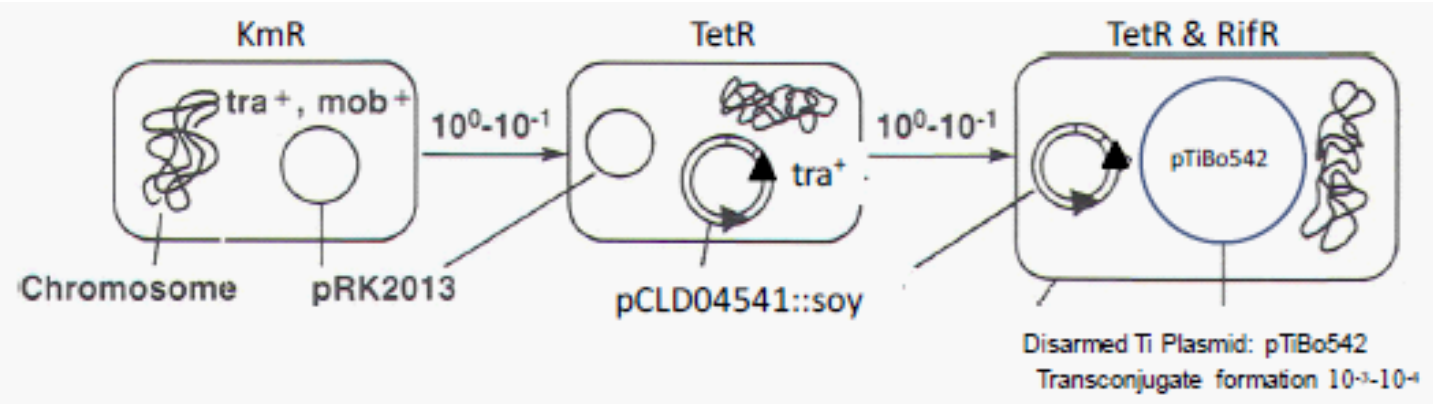

Figure 1. Triparental mating schema to mobilize soybean BACs into transformation competent Agrobacterium tumefaciens. 


\section{A. thaliana transformation}

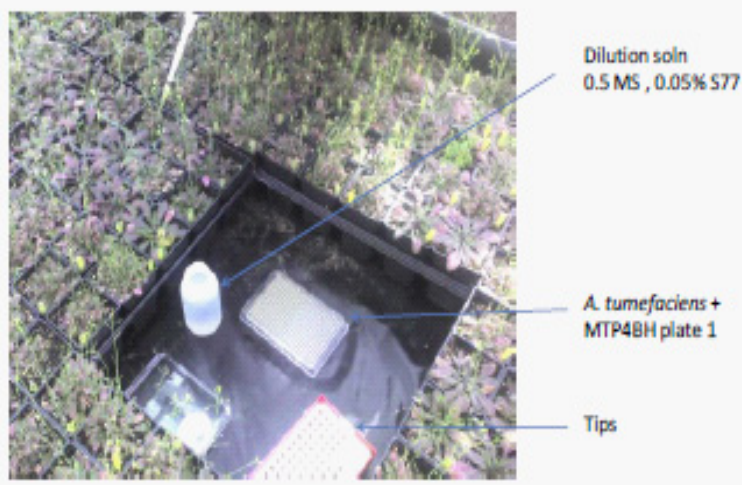

\section{Selection Schema}

\section{T0 flower transformation}

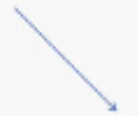

T1 Kanamycin selection $300 \mathrm{ug} / \mathrm{ml}, 3$

\author{
applications
}

T2 Line purification, Kanamycin selection $300 \mathrm{ug} / \mathrm{ml}$, on emergence

Figure 2. Transformation and selection of A. thaliana transgenic with BACs.

A.

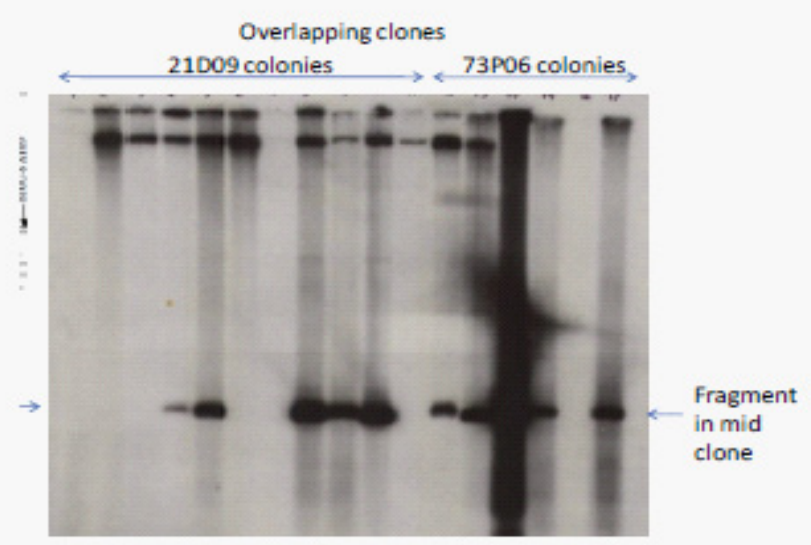

B

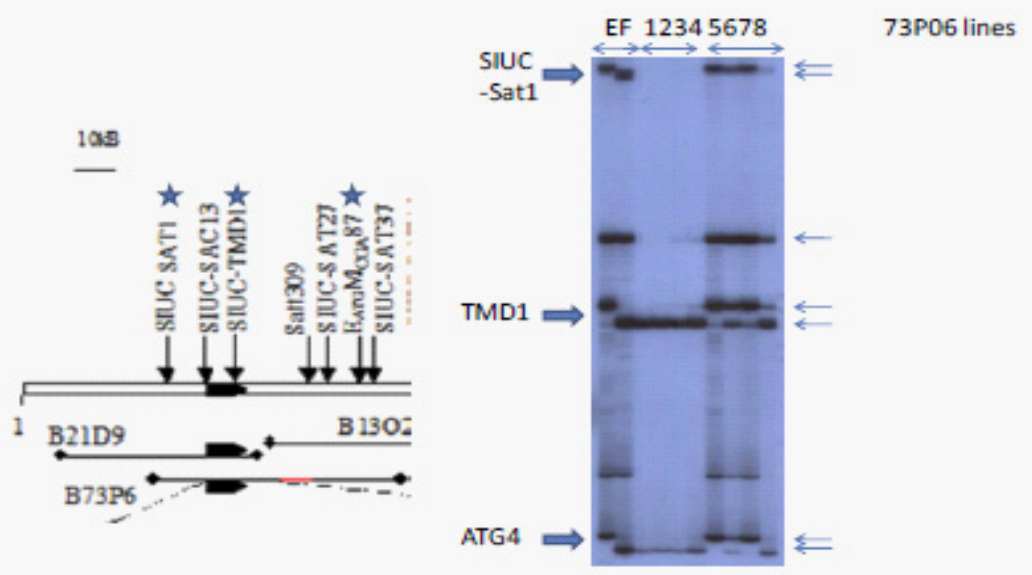

Figure 3. Hybridization of right border probes to single colony derived strains of A. tumefaciens (A) and PCR amplifications from single plants transgenic (B) with BAC clones derived from the rhg 1 region. Panel $A$ shows some clones have right border sequences and so are full length clones but about half do not and so are deletions. Panel $B$ shows an ideogram of the overlapping BAC clones B73P6 and PCR amplifications of three markers contained in the clone from $A$. thaliana's DNA extracted from transformed plants (lanes 1-4) compared to the alleles in Essex (E) and Forrest (F) soybean cultivars and 4 RILs (Lanes 5-8). 
Table 1. Some of the BACs, mutant and non-mutant soybean lines to be transformed for complementation.

\begin{tabular}{|c|c|c|c|c|}
\hline $\begin{array}{l}\text { SoyGD BIBAC Clone } \\
\text { names }\end{array}$ & Phenotypes & $\begin{array}{l}\text { Scafold } \\
\text { BIBAC }\end{array}$ & $\begin{array}{l}\text { Insert } \\
\text { Size kpb }\end{array}$ & Dominant? \\
\hline Gm-SIU1-B1 OOB 10 & Rhg 4 bigenic resistance to $\mathrm{SCN}$ a & B1 1 G08 & 140 & Yes \\
\hline Gm-SIU1-B73P06 & rhg 1 bigenic resistance to $\mathrm{SCN}$ and $\mathrm{Rfs} 2$ for SDS ${ }^{a}$ & B2704 & 79 & Co- \\
\hline Gm-SIU2-H050N07 & Rpg $1-b$ resistance to bacterial pustule ${ }^{b}$ & $\mathrm{H} 78 \mathrm{Cl} 4$ & 110 & Yes \\
\hline Gm-SIU1-B54E07 & $T$ Tawny Pubescence; Flavonoid-3-monooxygenase ${ }^{c}$ & $\mathrm{H} 62108$ & 82 & Yes \\
\hline Gm-SIU2-H40008 & W1 White Flower and Black Hila Color ${ }^{d}$ & H33M1 8 & 153 & No \\
\hline Gm-SIU2-H82C08 & Rfs 1 root resistance to SDS & H37G19/B4 & 130 & Yes \\
\hline Gm-SIU1-H49N1 4 & Rps 4 resistance to Phytophtora root rot & $\mathrm{H} 8 \mathrm{H} 15$ & 120 & Yes \\
\hline
\end{tabular}

a Rhg 4 and rhg 1 each encodes transmembrane receptor like kinase. Resistant and susceptible alleles differ by 3-6 amino acid changes and 23 base changes. There are mutant lines derived from Forrest. ${ }^{b} \mathrm{Rpg} 7 \mathrm{-b}$ encodes a nucleotide binding leucine rich repeat protein. ${ }^{c} \mathrm{~T}$ encodes flanonoid-3 monoxygenase (EC1.13.14.21). The recessive genes differ from the dominant by deletion of a single $C$ nucleotide. There are mutant lines. ${ }^{d}$ W encodes an unknown enzyme, probably a glycosidase. (Lightfoot, IJPG 2008:1-22. doi:10.1 155/2008/793158).

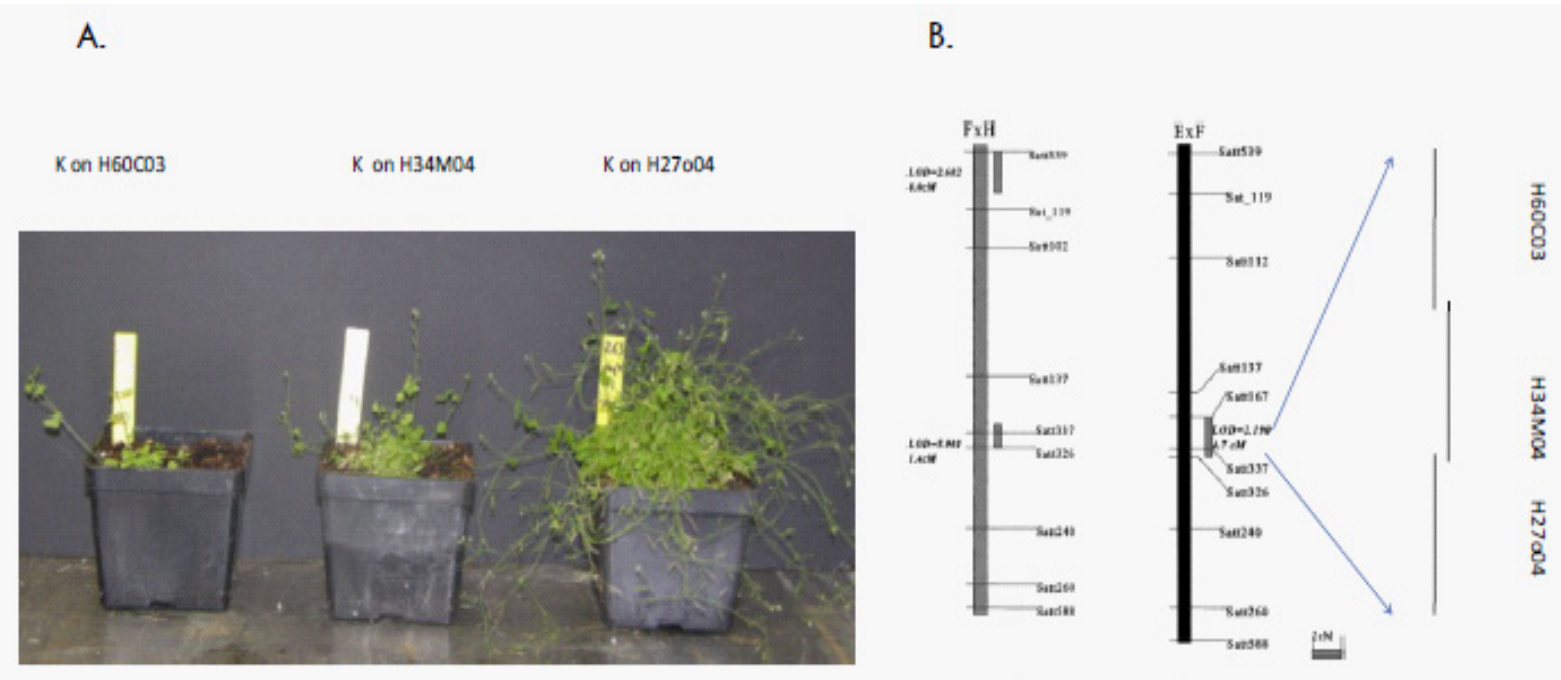

Figure 4. $B A C$ transformed $A$. thaliana with 3 clones from a region underlying soybean seed yield. Panel $A$ shows the differences in biomass, growth, and vigor among the lines. Panel B shows an ideogram of the QTL region and the BAC clones positions in that interval.

border and the multicloning site, causing the selectable marker to be the first gene transferred, This partial deletion series can be advantageous. With whole insert transformation Each phenotype may be associated with the 20-35 genes encoded by an individual BAC but with the deletion derivatives a phenotype might be associated with a smaller number of genes.

\section{Mutant Complementation Using Transformation}

A popular approach for the study of gene function is mutant complementation, which involves transformation of mutants with the wild alleles. Therefore, development of transformation protocols is an essential component of functional genomics research. In soybean, A. tumefaciens mediated transformation of cultured cells with Forrest BAC clones has been successfully achieved us- ing previously described protocols involving the T-DNA vector pCLD04541. In this protocol, npt II gene was used as a plant selectable marker, and kanamycin as used as a selective agent. Screen-able markers are available in some BAC clones (Table 1). Whole BAC transformation is important because fine maps locating loci at genetic distance of $0.25 \mathrm{cM}$ that is equivalent to $50-150 \mathrm{Kbp}$ were earlier prepared using RILs and NILs. The first set of clones selected for transformation are listed in Table 1 , and provided for complementation of easily score-able phenotypes in mutants. For instance, dominant mutant phenotypes of traits like pubescence, color and disease resistances should be evident in the very first products of transformation. BAC transformation with sets of overlapping clones will be the best approach in situations where an individual locus represents a cluster of genes or a large insertion/deletion (Ashfield et al., 2003; Tri- 


\section{Rpg1 on H050n07}

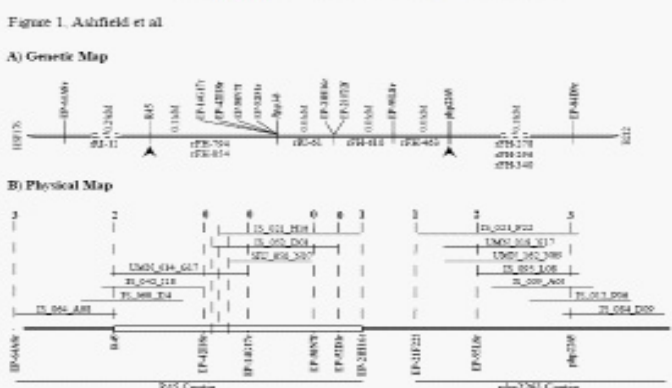

Disease R QTL Rpg1

F on HSONO7

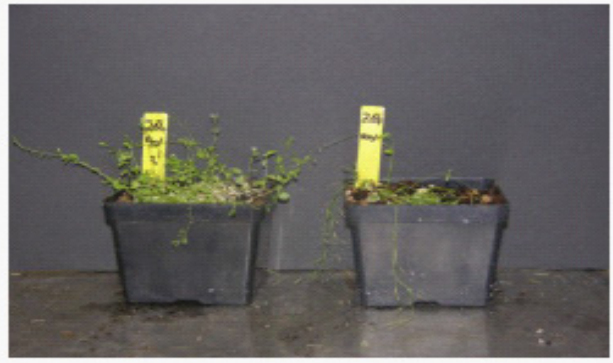

Figure 5. Transformation of A. thaliana with a BAC encompassing Rpg 1 caused spontaneous necrosis on the leaves that reduced seed yield. (A) The fine map of Rpg 1 showing the clone H50n07 encompassed part of the Rpg 1 locus (From Ashfield et al., 2003). (B) Two replicated transformation events selected at the Tl both showed the necrosis effect on leaves.

Rfs1 on B30M24

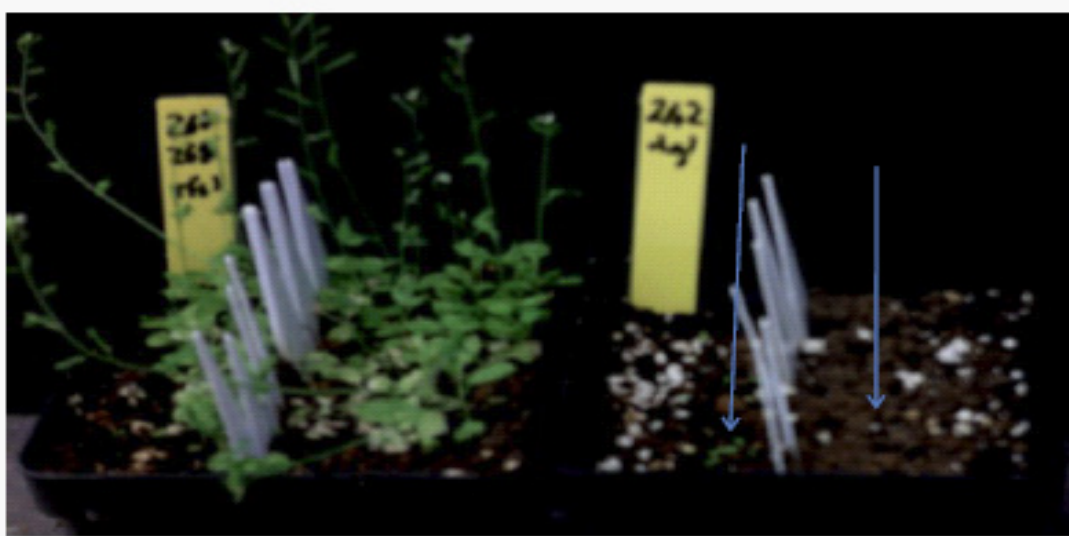

minus

plus

minus

plus

minus

Fusarium

Figure 6. Transformation of $A$. thaliana with a BAC encompassing Rhg $1 / R f s 2$ caused stunting that reduced seed yield. However, the clone bearing $R f s 2$ clone provided partial resistance to damping off by Fusarium virguliforme. Arrowed are stunted plants.

witayakorn et al., 2005; Bhattacharrya et al., 2005; Ruben et al., 2006; Afzal et al., 2008).

Phenotypes were found for clones predicted to underlie seed yield, oil content, root development, resistance to $\mathrm{SCN}$ and bacterial pustule. For seed yield the interval had be reduced to about $0.7 \mathrm{cM}$ or $300 \mathrm{kbp}$ by comparative mapping in two populations (Yuan et al., 2002). The three BACc transformed into A.thaliana gave three different phenotypes (Figure 4). The lines transgenic with $\mathrm{H} 27004$ were very high yielding giving 3 fold the seed of the average lines transformed at the same time in the same growth chamber. The lines transgenic with $\mathrm{H} 34 \mathrm{m0} 4$ were typical of the average. However, lines transformed with $\mathrm{H} 60 \mathrm{cO} 3$ were suppressed in yield four fold compared to lines grown at the same time in the same growth chamber. That two clones from the same QTL could have opposite effects was pre- dicted from fine mapping studies where QTL can often be separated into different loci. Loci of opposite effects on seed yield form part of the basis of hybrid vigor in outbreediing crops. However, in soybean they are undesirable and need to be separated by selection of recombination events that have positive yield effect in phase.

Disease resistance genes had effect on $A$. thaliana development in all three cases tested. With lines transgenic with the clone $\mathrm{H} 50 \mathrm{n} 07$ there was marked auto-necrosis (Figure 5). The genes encoded by the BAC clone include the NB-LRR proteins that underlie the resistance of soybean to bacterial pustule. The genes alone used as subclones do not cause the effect shown here (Ashfield et al., 2004; 2006) though they do provide A. thaliana resistance to Pseudomonas syringae pv. glycines. The whole BAC may cause the genes encoded be regulated in a distinct manner 


\section{A. The MTP of Soybean}

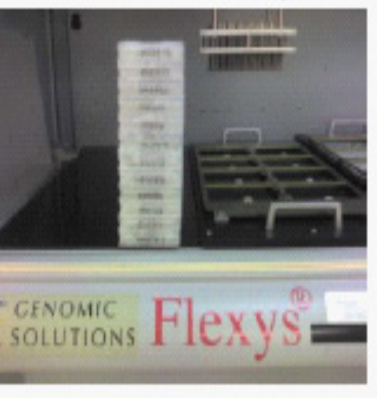

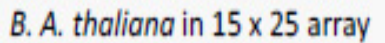

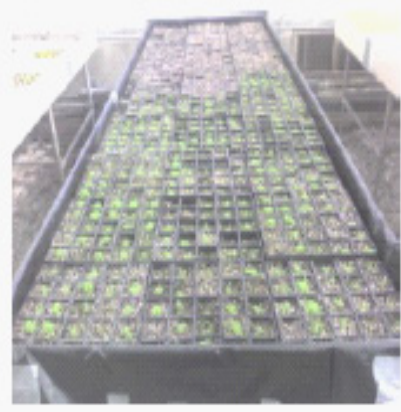

C. A. thaliana transformation

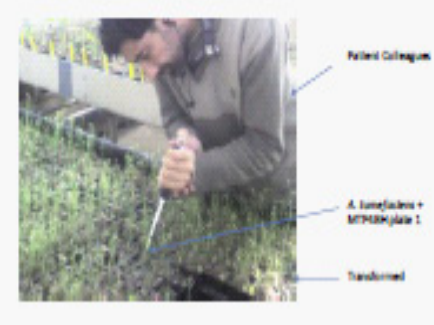

D. A. thaliana transformed pre shattering

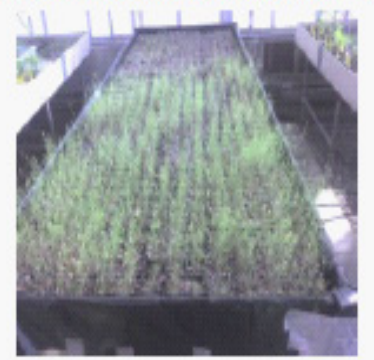

Figure 7. The process of transformation of the MTP of BAC clones into A thaliana. Panel A shows the use of a robot for triparental matings of the MTP of soybean. Panel B shows plants growing in a $15 \times 25$ array. Panel $C$ shows the transformation methos in action. Panel D shows the mature plants ready to harvest.

causing an inappropriate hypersensitive reaction.

In contrast to Rpgl the disease resistance loci on BACs encompassing $\mathrm{rhg} 1 / R f_{s} 2$ and $R f_{s} 1$ did not cause necrosis, so this is not an effect of all loci (Figure 6). The rhg 1 clones stunted plant development and produced a clavata like phenotype. The genes encoded by the BAC include a RLK in the same family as clavata 1. This protein does bind CLE domains, including that in nematode secretions. The Forrest allele of the rhg 1 locus has been implicated in reducing seedling root growth, germination and seedling vigor, serious negative agronomic effect of SCN resistance.The disease resistance loci on BACs encompassing Rfs 1 did not cause any negative effects and the vigor of plants was greater when challenged with Fusarium. The fine map of showing the clone 73 p06 encompassed part of the Rhgl locus (Figure 3) and B30m24 appeared to encompass part of the Rfs 1 locus.

There were no phenotypic changes from clones predicted to encode flower color, pubescence color or phytpophthora resistance. Clearly not all clones will have an effect on A. thaliana development. The BACs will be introducing 20- 30 new genes per line, but these will be regulated from endogenous promoter rather than overexpressed. It will be interesting to assay the extent of genetic perturbation in these lines by microarray.

\section{Massively Parallel Plant Transformation with BACs}

To date (mid 2012) the transformation of the entire MTP to Arabidopsis thaliana had reached the half was point. Five of the twelve 384 well plates of MTP4BH had been used to generated T2 seed. The efforts were limited by time and greenhouse space. Relatively few phenotypic effects were found in the T2 selections suggesting that the phenotype observed with mapped clones was the effect of enrichment.

\section{Conclusions}

The non-redundant part of the soybean genome can be transferred to Arabidopsis by minimum tile of 5,836 transformations. . Genes underlying known QTL identified for protein and oil on linkage group Al (Chromosome 5) were separated on two BACs. Seed yield QTL on linkage group K (Chromosome 9) could be divide into 3 BACs with opposite effects on growth. Two BACs from linkage group $G$ (chromosome 9) encode rhg 1 / Rfs 2 that stunts growth and Rfs 1 that resists Fusarium. The BAC encompassing disease resistance gene Rpgl appeared to cause auto-apopotosis..

Isolating genes underlying many QTL might be more efficient by mass transformation than fine mapping. There are a lot of QTL mapped in soybean, about 1,500 at the last count. Many more will be discovered by new mapping techniques. There are 79 traits and 307 QTL mapped in ExF92 alone (Lightfoot 2008). Fine mapping is very expensive and slow depending on the generation of massive populations to find rare recombinants. In contrast it takes about $40 \mathrm{BACs}$ to encompass $10 \mathrm{cM}$ (4 Mbp), the mean interval for a coarsely mapped QTL. Forty transformation for a thousand QTL would be a massive undertaking. Therefore, whole genome transformation with 5-6 thousand clones and development of an immortal seed bank will be more efficient than transformations QTL by QTL.

The en masse transformation method might be extended to soybean by hairy root transformation of composite traits. For whole soybean transformation kanR is a difficult selectable marker to rely upon. Therefore, a suicide plasmid was developed to introduce the part selectable marker to the right border region by recombination. The use of the plasmid will be reported.

\section{References}

Achenbach L, J Patrick, and L Gray (1996) Use of RAPD markers as a diagnostic tool for the identification of Fusarium solani isolates that cause soybean sudden death syndrome. Plant Dis 80: 1228-1232.

Afzal AJ and DA Lightfoot (2007) Soybean disease resistance protein RHG 1-LRR domain expressed, purified and refolded from Escherichia coli inclusion bodies: preparation for a functional analysis. Protein Expr Purif 53: 346-55.

Afzal J, A Wood, and DA Lightfoot (2008) Plant receptor-like serine threonine kinases: Roles in signaling and plant defense. Molecular Plant Microbe Interactions 21 : 507-517.

Anand SC (1992) Registration of "Hartwig" soybean. Crop Sci 32: 1060-1070. 
Aoki T, K O'Donnell, Y Homma, and AR Lattanzi (2003) Sudden-death syndrome of soybean is caused by two morphologically and phylogenetically distinct species within the Fusarium solani species complex F. virguliforme in North America and F. tucumaniae in South America. Mycologia 95: 660-684.

Ashfield T, A Bocian, D Held, A Henk, L Marek, D Danesh, S Penuela, K Meksem, DA Lightfoot, N Young, R Shoemaker, and T Innes (2003) Genetic and physical mapping of the soybean Rpg $1-b$ disease resistance gene reveals a complex locus containing several tightly linked families of NBS/LRR genes. Mol Plant Microbe Interact 16: 817-826.

Baker RA, and S Nemec (1994) Soybean sudden death syndrome: isolation and identification of a new phytotoxin from cultures of the causal agent, Fusarium solani. Phytopathology 84: 1144.

Chang SJC, TW Doubler, V Kilo, RJ Suttner, JH Klein, ME Schmidt, PT Gibson, and DA Lightfoot (1996) Two additional loci underlying durable field resistance to soybean sudden-death syndrome (SDS). Crop Sci 36: 1624-1628.

Chen NWG, Sévignac M, Ashfield T, Innes RW, Geffroy V (2010) Specific resistances against Pseudomonas syringae effectors AvrB and AvrRpm 1 have evolved differently in common bean, soybean, and Arabidopsis. New Phytologist 187: 941-956

Covert SF, T Aoki, K O'Donnell, D Starkey, A Holliday, DM Geiser, F Cheung, CD Town, A Strom, J Juba, M Scandiani, and XB Yang (2007) Sexual reproduction in the soybean sudden death syndrome pathogen Fusarium tucumaniae. Fungal Genetics and Biology 44: 799-807.

Farias-Neto AF, R Hashmi, ME Schmidt, SR Carlson, GL Hartman, S Li, RL Nelson, and BW Diers (2007) Mapping and confirmation of a sudden death syndrome resistance QTL on linkage group D2 from the soybean genotypes 'PI 567374' and 'Ripley'. Mol Breeding 20: 53-62.

Gibson PT, MA Shenaut, VN Njiti, RJ Suttner, and O Myers Jr (1994) Soybean varietal response to sudden death syndrome. p. 436-446. In D. Wilkinson. ed. Proc. Twenty-fourth Soybean Seed Res. Conf., Chicago, IL. 6-7 Dec. 1994. Am Seed Trade Assoc Washington DC.

Gray LE, LA Achenbach, RJ Duff, and DA Lightfoot (1999) Pathogenicity of Fusarium solani f. sp. glycines isolates on soybean and green bean plants. J Phytopathol 147: 281-284.

Hartman GL, YH Huang, RL Nelson, and GR Noel (1997) Germplasm evaluation of Glycine max for resistance to Fusarium solani, the causal organism of sudden death syndrome. Plant Dis $81: 515-518$.

Hashmi RY (2004) Inheritance of resistance to soybean sudden death syndrome. SDS. in 'Ripley' x 'Spencer' F5 derived lines. PhD Dissertation, Plant Biology, SIUC, Carbondale, USA.

Hnetkovsky N, SJC Chang, TW Doubler, PT Gibson, and DA Lightfoot (1996) Genetic mapping of loci underlying field resistance to soybean sudden death syndrome (SDS). Crop Sci 36: 393-400.

Iqbal MJ, K Meksem, VN Niiti, MA Kassem, and DA Lightfoot (2001) Microsatellite markers identity three additional quantitative trait loci for resistance to soybean sudden-death syndrome (SDS) in Essex $x$ Forrest RILs. Theor Appl Genet 102: 187-192.

Iqbal MJ, S Yaegashi, R Ahsan, KL Shopinski, and DA Lightfoot (2005) Root response to Fusarium solani f. sp. glycines: temporal accumulation of transcripts in partially resistant and susceptible soybean. Theor Appl Genet 1 10: 1429-1438.

Ji J, MP Scott, and MK Bhattacharyya (2006) Light is essential for degradation of ribulose-1,5-biphosphate carboxylase-oxygenase large subunit during sudden death syndrome development in soybean. Plant Biology 8: 597-605.

Jin H, GL Hartman, CD Nickell, and JM Widholm (1996) Characterization and purification of a phytotoxin produced by Fusarium solani, the causal agent of soybean sudden death syndrome. Phytopathol- ogy 86: 277-282.

Kassem MA, J Shultz, K Meksem, Y Cho, AJ Wood, MJ lqbal, and DA Lightfoot (2006) An Updated 'Essex' by 'Forrest' Linkage Map and First Composite Interval Map of QTL Underlying Six Soybean Traits. Theor Appl Genet 113: 1015-1026.

Kassem MA, K Meksem, AJ Wood, and DA Lightfoot (2007) Additional QTL for SDS and SCN resistances in a soybean recombinant inbred line population of 'Essex' by 'Forrest'. Reviews in Biology \& Biotechnology $6(1): 11-19$.

Kazi S (2005) Identification of twenty three loci conditioning agronomic traits in recombinant inbred and near isogeneic soybean lines derived from Flyer by Hartwig. MS Thesis SIUC Carbondale IL, USA, pp. 212.

Kazi S, J Shultz, R Bashir, J Afzal, VN Njiti, and DA Lightfoot (2008) Separate loci underlie resistance to soybean sudden death syndrome in 'Hartwig' by 'Flyer'. Theor and App Genet 116: 967-977.

Kazi S, VN Niiti, TW Doubler, J Yuan, MJ lqbal, S Cianzio, and DA Lightfoot (2007) Registration of the Flyer by Hartwig Recombinant Inbred Line Mapping Population. J Plant Registr 1: 175-178

Li S and GL Hartman (2003) Molecular detection of Fusarium solani f. sp. glycines in soybean roots and soil. Plant Pathol 52:74-78.

Lightfoot DA (2008) Soybean Genomics: Developments through the use of cultivar Forrest. International J of Plant Genomics 2008: 1-22. doi: $10.1155 / 2008 / 793158$.

Lozovaya VV, AV Lygin, OV Zernova, S Li, GL Hartman, and JM Widholm (2004) Isoflavonoid accumulation in soybean hairy roots upon treatment with Fusarium solani. Plant Physiol Biochem. 42: 671-679.

Lozovaya VV, AV Lygin, OV Zernova, S Li, JM Widholm, and GL Hartman (2005) Lignin degradation by Fusarium solani f. sp. glycines. Plant Disease 90: 77-82.

Meksem K, P Pantazopoulos, VN Njiti, DL Hyten, PR Arelli, and DA Lightfoot (2001) 'Forrest' resistance to the soybean cyst nematode is bigenic: saturation mapping of the Rhg 1 and Rhg 4 loci. Theor and App Genet 103: 710-717.

Meksem K, TW Doubler, K Chancharoenchai, VN Njiti, SJC Chang, PR Arelli, PB Cregan, LE Gray, PT Gibson, and DA Lightfoot (1999) Clustering among loci underlying soybean resistance to Fusarium solani, SDS and SCN in near-isogenic lines. Theor Appl Genet 99: 1131-1142.

Mueller DS, RL Nelson, GL Hartman, and WL Pederson (2003) Response of commercially developed soybean cultivars and ancestoral soybean lines to Fusarium solani f. sp. glycines, the causal organism of sudden death syndrome. Plant $D$ is $87: 827-831$.

Niiti VN and DA Lightfoot (2006) Genetic analysis infers Dt loci underlie resistance to SDS caused by Fusarium virguliforme in indeterminate soybeans. Can J Plant Science 41: 83-89.

Njiti VN, JE Johnson, TA Torto, LE Gray, and DA Lightfoot (2001) Inoculum rate influences selection for field resistance to soybean sudden death syndrome in the greenhouse. Crop Sci 41: 1726-1731.

Niiti VN, K Meksem, MJ Iqbal, JE Johnson, MA Kassem, KF Zobrist, VY Kilo, and DA Lightfoot (2002) Common loci underlie field resistance to soybean sudden death syndrome in Forrest, Pyramid, Essex, and Douglas. Theor Appl Genet 104: 294-300.

Niiti VN, L Gray, and DA Lightfoot (1997) Rate-reducing resistance to Fusarium solani f.sp. phaseoli [nee: glycines] underlies field resistance to soybean sudden-death syndrome. SDS). Crop Sci 37: 1-12

Niiti VN, MA Shenaut, RJ Sutter, ME Schmidt, and PT Gibson (1996) Soybean response to soybean sudden-death syndrome: inheritance influence by cyst nematode resistance in Pyramid $x$ Douglas progenies. Crop Sci 36: 1165-1170

Niiti VN, O Myers Jr, D Schroeder, and DA Lightfoot (2003) Roundup ready soybean: Glyphosate effects on Fusarium solani root colonization and sudden death syndrome. Agronomy J 95: 11 140-1 145. 
Niiti VN, TW Doubler, RJ Suttner, LE Gray, PT Gibson, and DA Lightfoot (1998) Resistance to soybean sudden death syndrome and root colonization by Fusarium solani f. sp. glycines in near-isogeneic lines. Crop Sci 38: 472-477.

O'Donnell K (2000) Molecular phylogeny of the Nectrai hematococccaFusarium solani species complex. Mycologia 92:919-938.

Prabhu RR, VN Niiti, JE Johnson, ME Schmidt, RJ Klein, and DA Lightfoot (1999) Selecting soybean cultivars for dual resistance to cyst nematode sudden death syndrome with two DNA markers. Crop Sci 39: 982-987.

Roy KW (1997) Fusarium solani on soybean roots: Nomenclature of the causal agent of sudden death syndrome and identity and relevance of F. solani form B. Plant Dis 81: 259-266.

Sanithchon J, A Vanavichit, S Chanprame, T Toojinda, T Triwitayakorn, VN Niiti, and P Srinives (2004) Identification of simple sequence repeat markers linked to sudden death syndrome resistance in soybean. Science Asia 30: 205-209.

Scherm H and XB Yang (1996) Development of sudden death syndrome of soybean in relation to soil temperature and soil water potential. Phytopathology 86: 642-649.

Schuster I, RV Abdelnoor, SRR Marin, VP Carvalho, AS Kiihl, JFV Silva, CS Sedyama, EG Barros, and MA Moreira (2001) Identification of a new major QTL associated with resistance to the soybean cyst nematode. Heterodera glycines). Theor Appl Genet 102: 91-96.

Shultz JL, CR Yesudas, S Yaegashi, JA Afzal, S Kazi, and DA Lightfoot (2006b) Three minimum tile paths from bacterial artificial chromosome libraries of the soybean. Glycine max cv. 'Forrest': Tools for structural and functional genomics. Plant Methods 2:9-18.

Shultz JL, D Kurunam, K Shopinski, MJ lqbal, S Kazi, KF Zobrist, R Bashir, $S$ Yaegashi, N Lavu, AJ Afzal, CR Yesudas, MA Kassem, C Wu, HB Zhang, CD Town, K Meksem, and DA Lightfoot (2006a) The Soybean Genome Database. SoyGD): A browser for display of duplicated, polyploid, regions and sequence tagged sites on the integrated physical and genetic maps of Glycine max. Nucleic Acids Res 34: D758-765.

Shultz JL, S Kazi, JA Afzal, R Bashir, and DA Lightfoot (2007) The development of $B A C$-end sequence-based microsatellite markers and placement in the physical and genetic maps of soybean. Theor Appl
Genet 114: 1081-1090.

Song QJ, LF Marek, RC Shoemaker, KG Lark, VC Concibido, X Delannay, JE Specht, and PB Cregan (2004) A new integrated genetic linkage map of the soybean. Theor Appl Genet 109: 122-128.

Srour A, AJ Afzal, N Saini, L Blahut-Beatty, N Hemmati, DH Simmonds, H El Shemy, CD Town, H Sharma, X Liv, W Li, and DA Lightfoot (2011) The receptor like kinase transgene from the $R h g 1 / R f s 2$ locus caused pleiotropic resistances to soybean cyst nematode and sudden death syndrome. BMC Genomics In Review.

Stephens PA, CD Nickell, and FL Kolb (1993) Genetic analysis of resistance to Fusarium solani in soybean. Crop Sci 33: 929-930.

Triwitayakorn K, VN Niiti, MJ lqbal, S Yaegashi, CD Town, and DA Lightfoot (2005) Genomic analysis of a region encompassing QRfs 1 and QRfs2: genes that underlie soybean resistance to sudden death syndrome. Genome 48: 125-138.

Ullah H, M Jasim, and DA Lightfoot (2012) Using a minimum tile path for plant transformations encompassing the entire soybean genome. Journal of Plant Genome Sciences 1 (2): XX-XX. doi: 10.5147/ ipgs.2011.0066.

Wrather JA, SR Kendig, SC Anand, TL Niblack, and GS Smith (1995) Effects of tillage, cultivar, and planting date on percentage of soybean leaves with symptoms of sudden death syndrome. Plant Dis 79:560-562.

Wrather JA, SR Koenning, and TR Anderson (2003) Effect of diseases on soybean yields in the United States and Ontario. 1999 to 2002. Plant Health Progr. online doi 10.1049.

Wrather JA, TR Anderson, DM Arsyad, J Gai, DL Ploper, A Portapuglia, HH Ram, JT Yorinori (1996) Soybean disease loss estimates for the top ten producing countries during. Plant Disease 79:107-1 10.

Yuan J, M Zhu, DA Lightfoot, MJ lqbal, JY Yang, and K Meksem (2008) In silico comparison of transcript abundances during Arabidopsis thaliana and Glycine max resistance to Fusarium virguliforme. BMC Genomics. 2008 2: S6.

Yuan J, VN Niiti, K Meksem, MJ lqbal, K Triwitayakorn, MA Kassem, GT Davis, ME Schmidt, and DA Lightfoot (2002) Quantitative trait loci in two soybean recombinant inbred line populations segregating for yield and disease resistance. Crop Sci 42: 271-277. 